(W)

Check for

Cite as

Nano-Micro Lett.

(2021) 13:57

Received: 23 September 2020

Accepted: 1 December 2020

Published online: 16 January 2021

(C) The Author(s) 2021

\section{Electrospinning of Flexible Poly(vinyl alcohol)/ MXene Nanofiber-Based Humidity Sensor Self-Powered by Monolayer Molybdenum Diselenide Piezoelectric Nanogenerator}

\author{
Dongyue Wang ${ }^{1}$, Dongzhi Zhang ${ }^{1}{ }^{凶}$, Peng $\mathrm{Li}^{2}{ }^{凶}$, Zhimin Yang $^{1}$, Qian $\mathrm{Mi}^{1}$, \\ Liandong $\mathrm{Yu}^{1} \bowtie$
}

\title{
HIGHLIGHTS
}

- A flexible piezoelectric nanogenerator (PENG) based on 2D single-layer $\mathrm{MoSe}_{2}$ flake on polyethylene terephthalate was fabricated.

- A high-performance flexible poly(vinyl alcohol)/MXene (PVA/MXene)-based humidity sensor was fabricated by electrospinning.

- The PVA/MXene composite-based humidity sensor was self-powered by MoSe ${ }_{2}$ PENG and exhibited excellent properties.

\begin{abstract}
Two-dimensional material has been widely investigated for potential applications in sensor and flexible electronics. In this work, a self-powered flexible humidity sensing device based on poly(vinyl alcohol)/ $\mathrm{Ti}_{3} \mathrm{C}_{2} \mathrm{~T}_{\mathrm{x}}$ (PVA/ MXene) nanofibers film and monolayer molybdenum diselenide $\left(\mathrm{MoSe}_{2}\right)$ piezoelectric nanogenerator (PENG) was reported for the first time. The monolayer

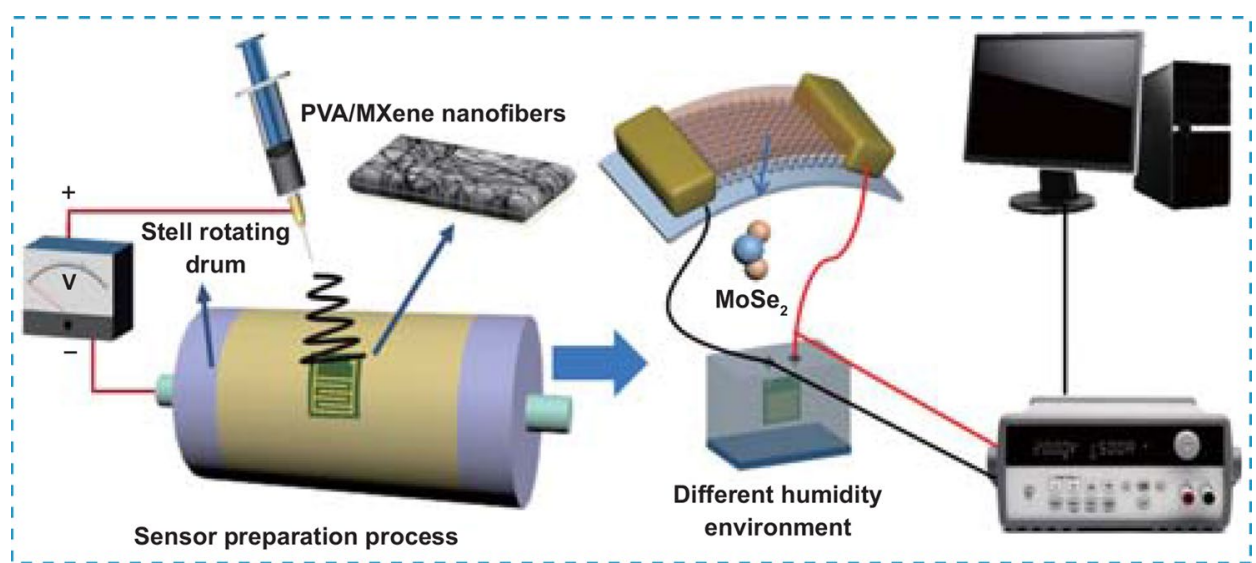
$\mathrm{MoSe}_{2}$-based PENG was fabri-

cated by atmospheric pressure chemical vapor deposition techniques, which can generate a peak output of $35 \mathrm{mV}$ and a power density of $42 \mathrm{~mW} \mathrm{~m}^{-2}$. The flexible PENG integrated on polyethylene terephthalate (PET) substrate can harvest energy generated by different parts of human body and exhibit great application prospects in wearable devices. The electrospinned PVA/MXene nanofiber-based humidity sensor with flexible PET substrate under the driven of monolayer $\mathrm{MoSe}_{2}$ PENG, shows high response of $\sim 40$, fast response/recovery time of $0.9 / 6.3 \mathrm{~s}$, low hysteresis of $1.8 \%$ and excellent repeatability. The self-powered flexible humidity sensor yields the capability of detecting human skin moisture and ambient humidity. This work provides a pathway to explore the high-performance humidity sensor integrated with PENG for the self-powered flexible electronic devices.
\end{abstract}

KEYWORDS Self-powered sensing; Monolayer molybdenum diselenide; Piezoelectric nanogenerator; Humidity sensor; Flexible electronics

Dongzhi Zhang, dzzhang@upc.edu.cn; Peng Li, pengli@mail.tsinghua.edu.cn; Liandong Yu, liandongyu@upc.edu.cn

1 College of Control Science and Engineering, China University of Petroleum (East China), Qingdao 266580, People's Republic of China

2 State Key Laboratory of Precision Measurement Technology and Instruments, Department of Precision Instruments, Tsinghua University,

Beijing 100084, People's Republic of China 


\section{Introduction}

Humidity sensor has become increasingly indispensable in many areas such as industrial manufacture, medical health, and air quality monitoring, especially in the Internet of Things and flexible electronics [1]. At present, many kinds of signal detection technique have been developed and applied to humidity detection, including capacitance [2], quartz crystal microbalance [3], bulk acoustic wave (BAW) [4], and surface acoustic wave (SAW) [5]. However, these detection techniques all require complex detection equipment and power source, which increases application cost and energy consumption. The traditional method is using the batteries to drive humidity sensors to work, which limits the wide range of application for sensors in Internet of Things. Usually, a small amount of power can make most types of sensors operate [6]. In view of the large amount of available clean energy existing in the surrounding environment or human body, we can harvest these energies to build sustaining self-powered sensing system [7-9]. Therefore, it is expected to develop a simple and low-cost humidity sensing system without external power supply through self-powered technology $[10,11]$

The latest technologies for collecting energy mainly include piezoelectricity [12, 13], triboelectricity [14-17], pyroelectricity [18], photoelectricity [19], and electromagnetism [20]. Among these technologies, nanogenerator based on triboelectricity and piezoelectricity is considered to have excellent application prospects due to its high durability and mechanical stability, especially in the field of self-powered sensors [21]. A piezoelectric nanogenerator (PENG) prepared from zinc oxide nanowires was first reported by Wang et al. in 2006, which gained great concern because of its excellent piezoelectric performance [22]. Liu et al. designed a self-powered multifunctional monitoring system using hybrid integrated triboelectric and piezoelectric microsensors, which can effectively monitor the relative humidity (RH) level and carbon dioxide concentration [23]. Zhang et al. reported a novel self-recovering triboelectric nanogenerator (TENG) as an active multifunctional sensor. The device has a wide humidity detection range $(20 \%-100 \% \mathrm{RH})$ and rapid response/recovery time $(18 / 80 \mathrm{~ms})$ [24]. Xia et al. designed a conductive copper tape-based TENG combined with $\mathrm{LiCl}$ for humidity detection. The TENG has a power density of $240.1 \mu \mathrm{W} \mathrm{cm}{ }^{-2}$, and the $\mathrm{RH}$ can be represented by the brightness of the LEDs driven by the TENG [25]. Tai et al. developed an air-driven triboelectric nanogenerator based on Ce-doped $\mathrm{ZnO}$-PANI, which was used to detect the $\mathrm{NH}_{3}$ concentration, flow rate, and frequency of exhaled gas [26]. Many new nanomaterials with excellent piezoelectric properties were continuously studied. Monolayer boron nitride (BN), $\mathrm{MoS}_{2}, \mathrm{MoSe}_{2}, \mathrm{WTe}_{2}, \mathrm{WSe}_{2}$, and $\mathrm{MoTe}_{2}$ have been theoretically predicted to exhibit piezoelectric property [27]. And it has been experimentally confirmed that single-layer $\mathrm{MoS}_{2}$ showed piezoelectric effect and was applied to PENG [28, 29].

In recent years, two-dimensional (2D) nanomaterials such as graphene, metal oxides, transition metal dichalcogenides (TMDs), metal organic frameworks (MOFs), black phosphorus have attracted tremendous interests due to their excellent physical, chemical, and electrical properties. Especially, 2D nanomaterials have been employed in constructing high-performance sensors and flexible electronic devices [30, 31]. In 2011, MXene was first synthesized by Yury et al. [32]. As a new 2D nanomaterial, MXene exhibits high specific surface area, high conductivity, and excellent flexibility, which is considered to have great application prospects in humidity and wearable sensors. Lu et al. found alkalized MXene exhibited much better sensing properties toward $\mathrm{NH}_{3}$ and humidity, compared with untreated MXene [33]. Due to excellent metal conductivity and hydrophilicity, MXene not only exhibits more excellent gas and humidity sensing properties, but also a very promising material for building flexible sensors to provide excellent mechanical stimulus sensing performance. Wang et al. reported a piezoresistive flexible sensor prepared by MXene/natural microcapsule, which has a fast response time (14 ms), satisfactory repeatability, and stability [34].

In this work, a self-powered flexible humidity sensor based on electrospinned poly (vinyl alcohol) $/ \mathrm{Ti}_{3} \mathrm{C}_{2} \mathrm{~T}_{\mathrm{x}}$ (PVA/ MXene) nanofibers film and monolayer molybdenum diselenide $\left(\mathrm{MoSe}_{2}\right)$ piezoelectric nanogenerator was reported for the first time. The monolayer $\mathrm{MoSe}_{2}$ PENG was fabricated on a flexible polyethylene terephthalate (PET) substrate. The PVA/MXene nanofibers film was prepared on the interdigital electrodes (IDEs) as the humidity-sensitive material through electrospinning technology. The prepared self-powered piezoelectric humidity sensor (PEHS) was driven by the monolayer $\mathrm{MoSe}_{2}$ PENG via converting mechanical energy to electric energy. The self-powered PVA/MXene nanofibers 
film humidity sensor has a large response, fast response/ recovery time, low hysteresis, and excellent repeatability. Furthermore, the humidity sensing mechanism of PVA/ MXene sensor was explored.

\section{Experiment}

\subsection{Materials}

Hydrochloric acid ( $\mathrm{HCl}$, analytical purity) and lithium fluoride (LiF, 99\%) were purchased from Sinopharm
Chemical Reagent. Titanium aluminum carbide powders $\left(\mathrm{Ti}_{3} \mathrm{AlC}_{2}\right)$ and poly (vinyl alcohol) (PVA) were from Shanghai Macklin Biochemical Technology.

\subsection{Materials Synthesis}

Synthesis of MXene: $\mathrm{LiF}(1 \mathrm{~g})$ was dispersed in a polypropylene plastic bottle with $6 \mathrm{M} \mathrm{HCl}$ solution $(20 \mathrm{~mL})$ and then was stirred for $5 \mathrm{~min}$ to ensure a fully dissolution. One gram of $\mathrm{Ti}_{3} \mathrm{AlC}_{2}$ was added slowly to the mixed solution to avoid violent reaction of the solution, followed by placed at

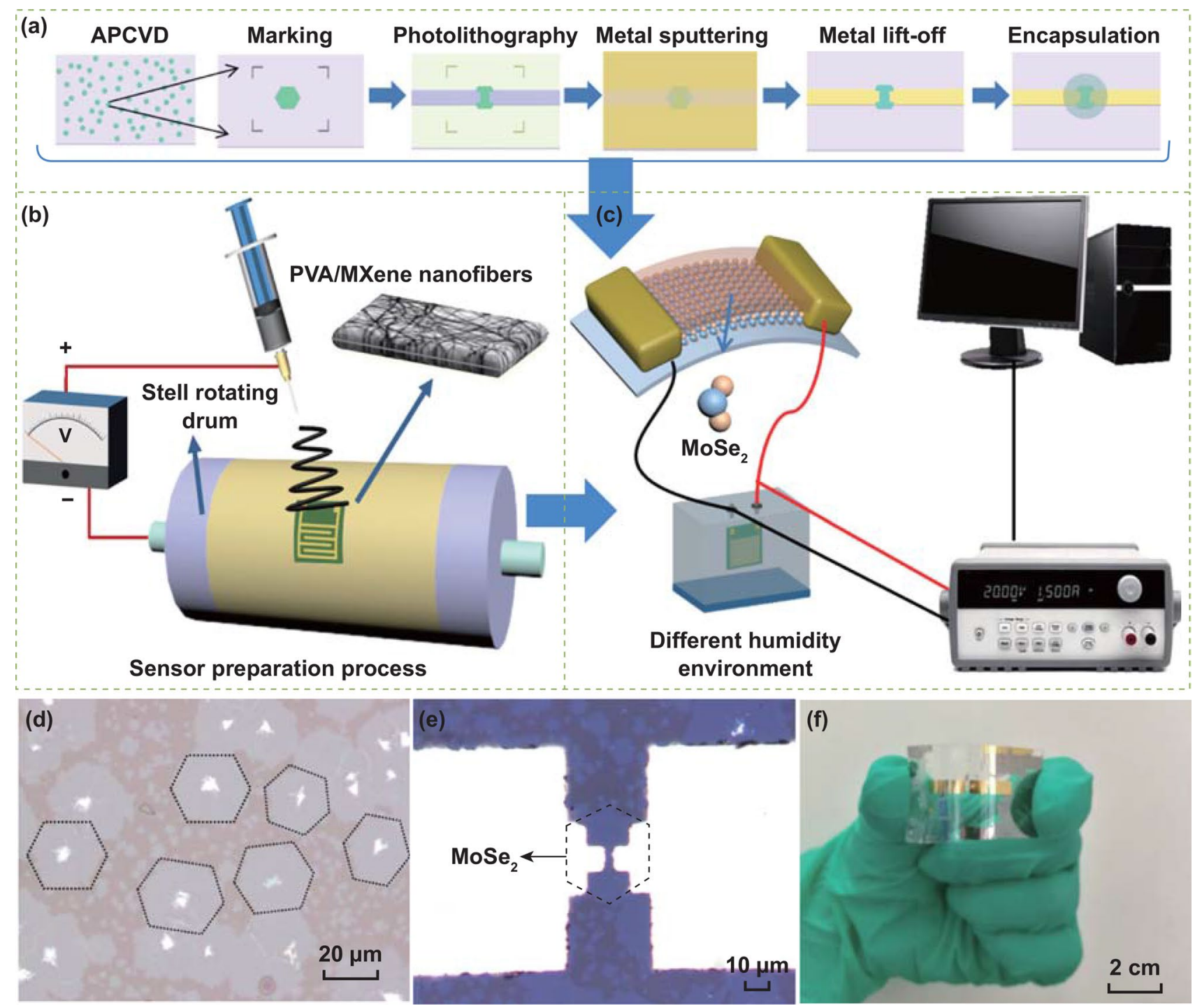

Fig. 1 Schematic diagram for the fabrication of a MoSe $e_{2}$-flake-based PENG and b PVA/MXene humidity sensor. c Schematic of the experimental platform for humidity sensing measurement. $\mathbf{d}$ Monolayer $\mathrm{MoSe}_{2}$ prepared by APCVD. e Optical microscope image of a MoSe $\mathrm{Siezoelectric}$ device with two electrodes. $\mathbf{f}$ Photograph of the flexible PENG device 
$35{ }^{\circ} \mathrm{C}$ for $24 \mathrm{~h}$. The product after reaction was washed with deionized water several times until the $\mathrm{pH}$ of supernatant is greater than or equal to 6 . And then, the product was treated via centrifugation at $3500 \mathrm{rpm}$ for $5 \mathrm{~min}$, and the dark green supernatant collected as delaminated $\mathrm{Ti}_{3} \mathrm{C}_{2} \mathrm{~T}_{\mathrm{x}}$.

Preparation of PVA/ $\mathrm{Ti}_{3} \mathrm{C}_{2} \mathrm{~T}_{\mathrm{x}}$ mixed suspensions: Electrospinning is a typical fiber manufacturing process that can produce fibrous nanofilms with larger specific surface area $[35,36]$. One gram of PVA was placed in $9 \mathrm{~g}$ of deionized water to obtain $10 \%$ (w/w) PVA solution. The solution was stirred for $3 \mathrm{~h}$ at $90{ }^{\circ} \mathrm{C}$. Then, the PVA/ MXene solution was prepared by adding $0.1 \mathrm{~g}$ of $\mathrm{Ti}_{3} \mathrm{C}_{2} \mathrm{~T}_{\mathrm{x}}$ into PVA solution and magnetic stirring for about $0.5 \mathrm{~h}$.

\subsection{Fabrication of the PENG and PEHS}

Monolayer $\mathrm{MoSe}_{2}$ flake was employed for the fabrication of the PENG. Figure 1a shows the schematic diagram for the fabrication of PENG. The electrodes were prepared by photolithography, metal deposition (10 nm Cr/100 nm Au), and lift-off process. As shown in Fig. 1d, e, the monolayer $\mathrm{MoSe}_{2}$ prepared by atmospheric pressure chemical vapor deposition (APCVD) method was transferred to flexible PET substrate and exhibits irregular hexagon, followed by packaging with polydimethylsiloxane (PDMS) film. The white dots in the middle are the centers of the crystal nucleus. The monolayer $\mathrm{MoSe}_{2}$ on PET is sealed by PDMS film, which is isolated from the external environment to avoid the influence of environment humidity. Figure 1f shows the photograph of the flexible PENG device. The monolayer $\mathrm{MoSe}_{2}$ flakes connected with Au electrodes along with armchair are performed. And the $\mathrm{MoSe}_{2}$ atomic orientation was identified by optical second harmonic generation (SHG) as in Fig. S1.

As shown in Fig. 1b, the humidity sensor with PVA/ MXene nanofibers film was prepared on interdigital electrodes (IDEs) with epoxy substrate using electrospinning technology. The dimension of IDEs is $8 \times 8 \mathrm{~mm}^{2}$, and the thickness is $0.3 \mathrm{~mm}$. Electrospinning is widely used for preparing continuous nanofibers from viscoelastic fluids through electrostatic repulsion force between surface charges. Electrospinning nanofibers have the advantages of small porosity, high porosity, and large specific surface area, and are promising building blocks for the fabrication of sensors. The applied voltage between positive and negative poles was $18 \mathrm{kV}$, and the needle-to-collector distance was $15 \mathrm{~cm}$. The flow rate is $0.3 \mathrm{~mL} \mathrm{~h}^{-1}$, and the duration is $0.5 \mathrm{~h}$. Schematic of the experimental platform for humidity sensing measurement is shown in Fig. 1c. PENG is driven by a tensile testing machine (MIT-1021). The humidity sensor was driven by PENG via converting mechanical energy into electrical energy. The humidity sensing properties were systematically investigated under a humidity range of 11-97\% $\mathrm{RH}$ at $25{ }^{\circ} \mathrm{C}$. The output voltage of PENG and PEHS was measured by a digital multimeter (Keysight 34470A).

\subsection{Characterization Instrument}

The structure and surface morphology of the PVA/MXene nanofibers were characterized using scanning electron microscopy (SEM, Hitachi S-4800, Japan) and transmission electron microscope (TEM, Jeoljem-2100, Japan). The X-ray diffractometer (XRD, Rigaku Miniflex 600) with $\mathrm{CuK} \alpha$ radiation $(\lambda=0.15418 \mathrm{~nm})$ was used to investigate its crystal structure. The $\mathrm{MoSe}_{2}$ atomic layer was characterized by confocal Raman microscopy (Horiba HR-800) with a laser wavelength of $514 \mathrm{~nm}$. Fourier transform infrared spectroscopy (FTIR) spectra were recorded using a PerkinElmer Spectrum Two FTIR spectrometer.

\section{Results and Discussion}

\subsection{Fundamental Measurement of PENG}

The thickness of monolayer $\mathrm{MoSe}_{2}$ was identified by AFM. Figure 2a shows the AFM topographic image of the hexagon-like-shaped monolayer $\mathrm{MoSe}_{2}$ flake, and Fig. 2b exhibits the corresponding height profile, which is about $0.8 \mathrm{~nm}$ for the monolayer $\mathrm{MoSe}_{2}$ structure [37]. Figure 2c shows the characterization result using Raman spectrum. The prepared $\mathrm{MoSe}_{2}$ has two major characteristic peaks at 239.7 and $289.7 \mathrm{~cm}^{-1}$, which are corresponding to the out-of-plane $\mathrm{A}_{1 \mathrm{~g}}$ and in-plane $\mathrm{E}_{2 \mathrm{~g}}$ modes, respectively [38]. Figure $2 \mathrm{~d}$ shows the operation scheme of the $\mathrm{MoSe}_{2}$ piezoelectric device. No induced charge was generated at both ends of the $\mathrm{MoSe}_{2}$ flake in the initial state. When the PENG was stretched, a monolayer of $\mathrm{MoSe}_{2}$ would generate charges with opposite polarities at its edges. When the PENG was released, reverse electron flow resulted in a negative voltage peak. Periodic 
(a)

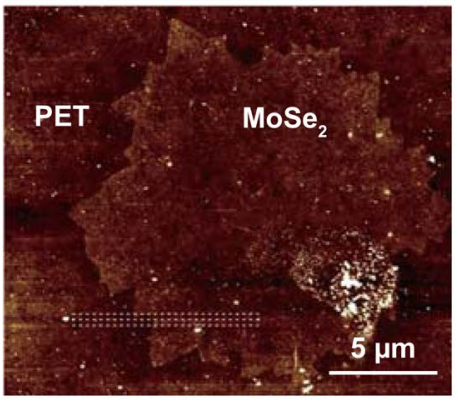

(d)

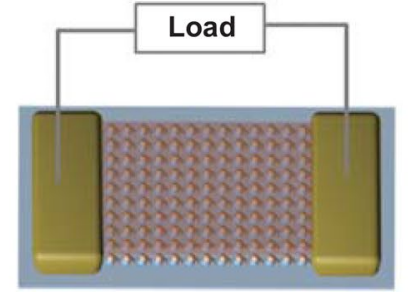

Initial state
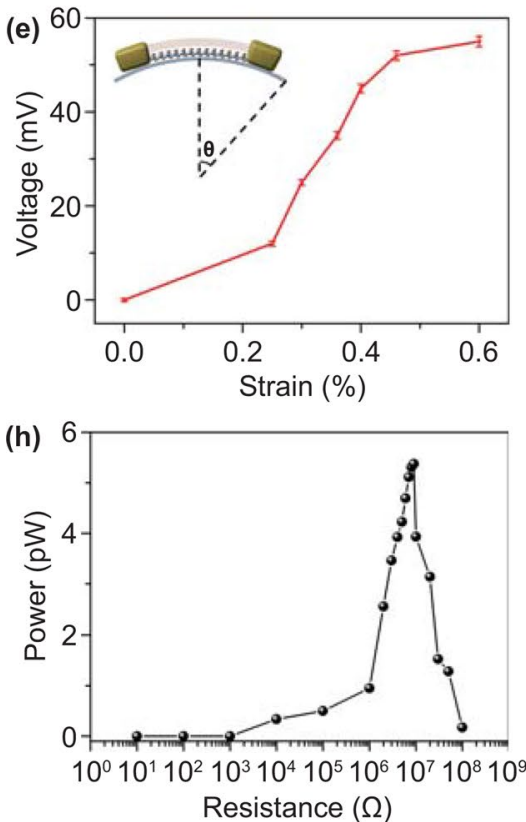
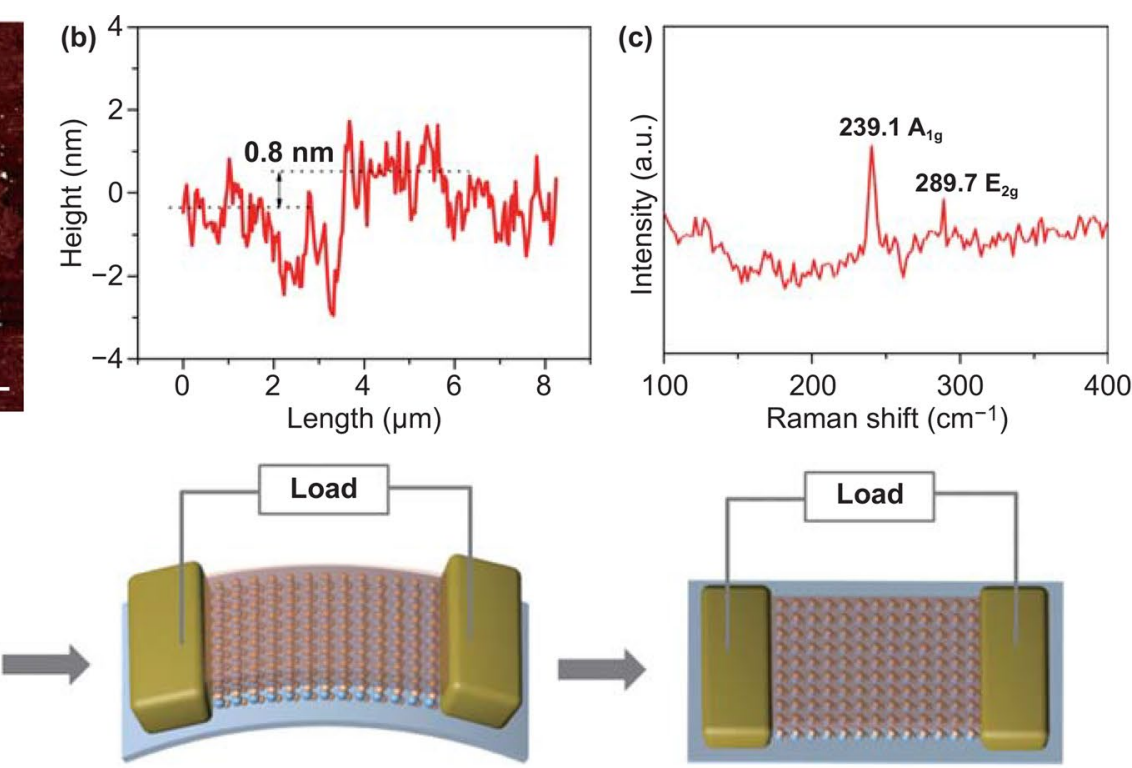

Stretched
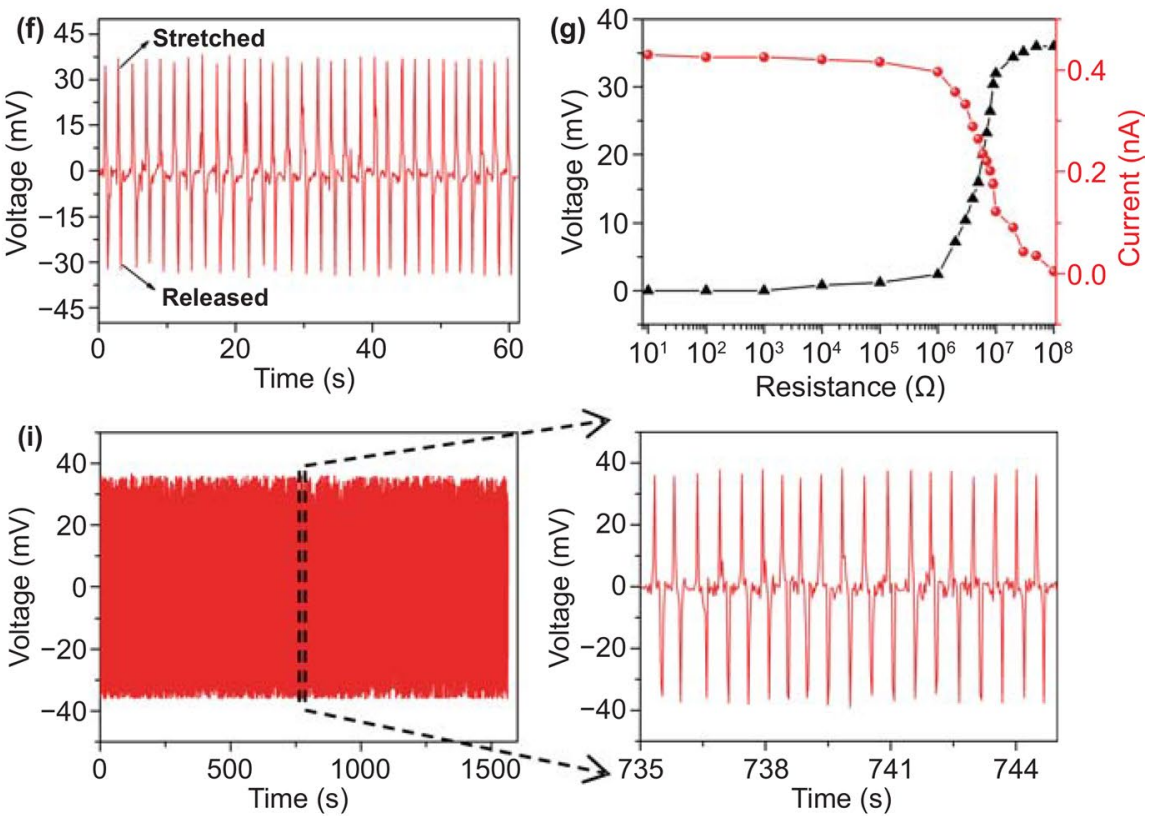

Fig. 2 a AFM topographic image of the single-layer $\mathrm{MoSe}_{2}$ flake. b Relative heights along the white lines in Fig. 2 a. $\mathbf{c}$ Raman spectrum of the monolayer $\mathrm{MoSe}_{2}$ flake. d Operation scheme of the monolayer MoSe ${ }_{2}$ piezoelectric device. e Open-circuit voltages of the device as a function of strain. f Real-time output voltage under $0.36 \%$ strain at a frequency of $0.5 \mathrm{~Hz}$. $\mathrm{g}$ Dependence of output voltage and current from a monolayer $\mathrm{MoSe}_{2}$ device under $0.36 \%$ strain as a function of external loading resistance. h Power versus the loading resistance. $\mathbf{i}$ Cyclic test showing the stability of monolayer $\mathrm{MoSe}_{2}$ device for prolonged period

stretching and releasing can cause PENG produces alternating positive and negative voltage output signals (Video S1). The piezoelectric output of a $\mathrm{MoSe}_{2}$ PENG is related to the magnitude of the applied strain. Figure 2e shows the relationship between open-circuit voltages and the strain of the device. The strain was defined as Eq. (1) [29]: $\varepsilon=\frac{h}{2 R}$

where $h$ is the thickness of the flexible substrate, $R$ is the radius of curvature when the PENG was stretched. It can be found that the output voltage rises as the degree of the applied strain increases. The output voltage can reach $55 \mathrm{mV}$ 
Table 1 Performance of the $\mathrm{MoSe}_{2}$ PENG in this work compared with the previous work

\begin{tabular}{lllll}
\hline Piezoelectric material & Open-circuit voltage $(\mathrm{mV})$ & Short-circuit current $(\mathrm{nA})$ & Power density $\left(\mathrm{mW} \mathrm{m}^{-2}\right)$ & Refs. \\
\hline ZnO NRs & 2.8 & 8500 & 0.08 & $\sim$ \\
PVDF-TrFE & 17 & $\sim$ & 1.14 & {$[39]$} \\
$\mathrm{SnS}_{2}$ nanosheet & 33 & 0.18 & 0.16 & {$[40]$} \\
$\mathrm{KNN}_{\text {piezo-resin/CFRP }}$ & 2 & $\sim$ & 2 & {$[41]$} \\
$\mathrm{MoS}_{2}$ nanosheet & 15 & 0.02 & 42 & {$[42]$} \\
$\mathrm{MoSe}_{2}$ nanosheet & 35 & 0.4 & This paper \\
\hline
\end{tabular}
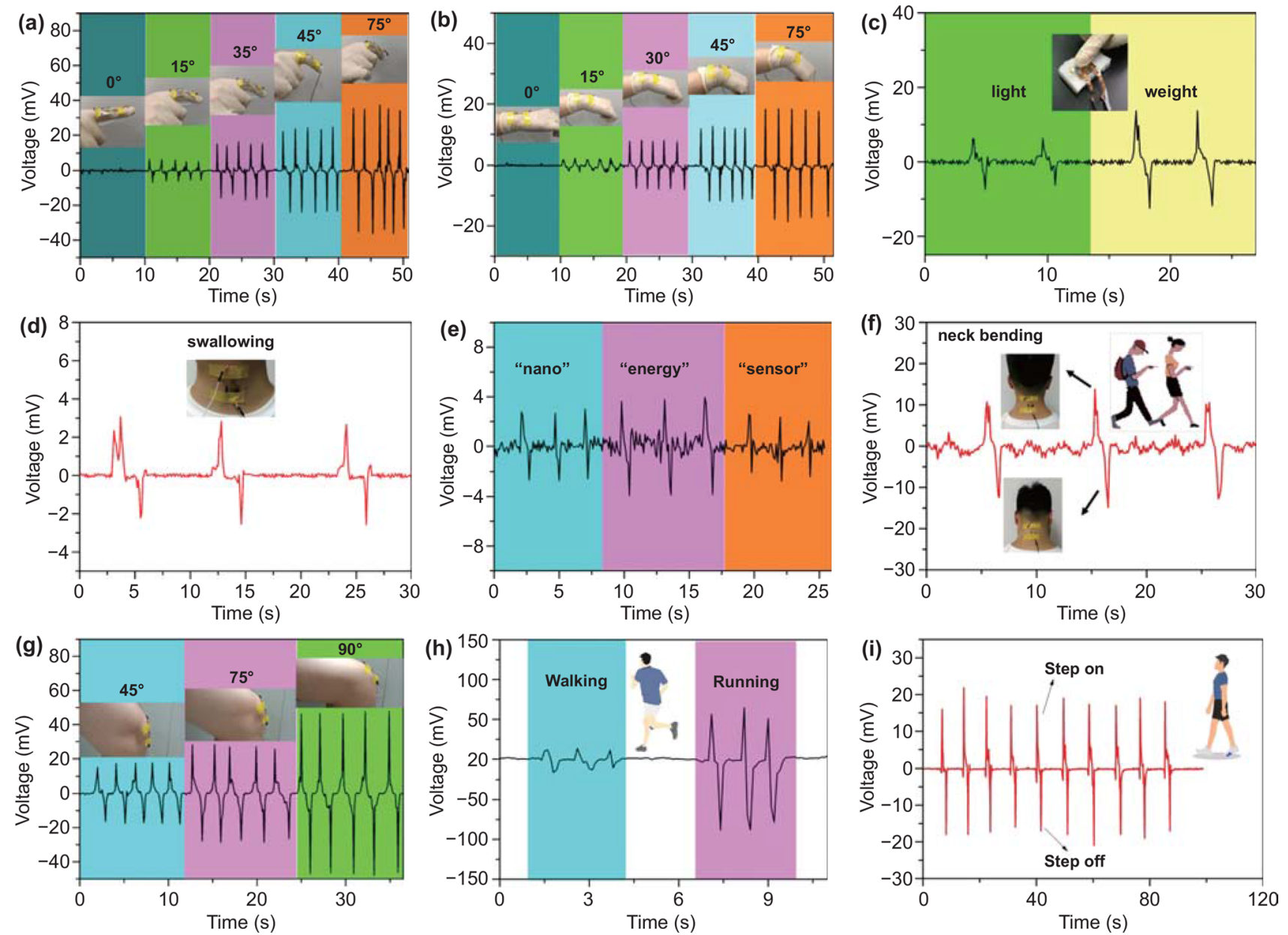

Fig. 3 Energy harvesting and application of $\mathrm{MoSe}_{2}$ device on various parts of the human body a Index finger joint. B Wrist. c Finger press. d Throat. e Output voltage change of flexible PENG in terms of various sound stimuli, such as "nano," "energy," and "sensor." $\mathbf{f}$ Neck bending. $\mathbf{g}$ Knee bending. h, i Human motion detection

at strain of $0.6 \%$, which is much higher than monolayer $\mathrm{MoS}_{2}$ PENG [28]. Figure $2 \mathrm{f}$ shows the real-time output voltage $(35 \mathrm{mV})$ under $0.36 \%$ strain at a frequency of $0.5 \mathrm{~Hz}$. As shown in Fig. S2, PENG is bent at different frequencies. The bending frequency has little effect on the voltage output of PENG. In subsequent experiments, we apply strain of $0.36 \%$ to avoid $\mathrm{MoSe}_{2}$ slippage. 
Figure $2 \mathrm{~g}$ shows the output voltage and current from the PENG at $0.36 \%$ strain under different loading resistances. The output current decreases slowly in low resistance range $(1 \Omega-100 \mathrm{M} \Omega)$ and then declines rapidly with the increasing load, and the voltage changes with the opposite trend. Figure $2 \mathrm{~h}$ shows the output power under different loading resistances. The maximal output power of PENG is up to $5.37 \times 10^{-9} \mathrm{~mW}$ at a loading resistance of $9.2 \mathrm{M} \Omega$ and a power density of about $42 \mathrm{~mW} \mathrm{~m}^{-2}$, which is higher than other kinds of PENGs (Table 1) [28, 39-42]. As shown in Fig. 2i, the cyclic test shows the good stability of PENG for prolonged period and indicates that the energy conversion is stable.

\subsection{Energy Harvesting of Human Activities}

We further explored the energy harvesting of $\mathrm{MoSe}_{2}$ device on various parts of the human body. Figure 3 a illustrates the energy harvesting at the knuckles (Video S2). The device is attached to the finger joint and bends with the finger. We can observe that the PENG generates different output voltages for wrist bending motions at five different angles of $0^{\circ}$,
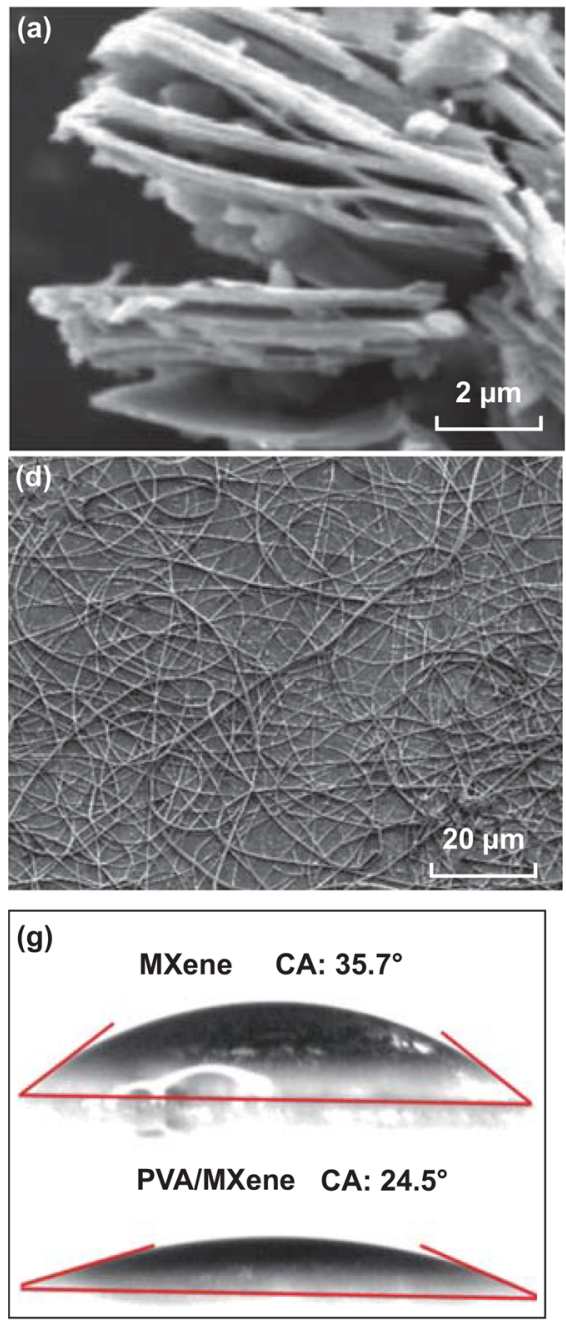
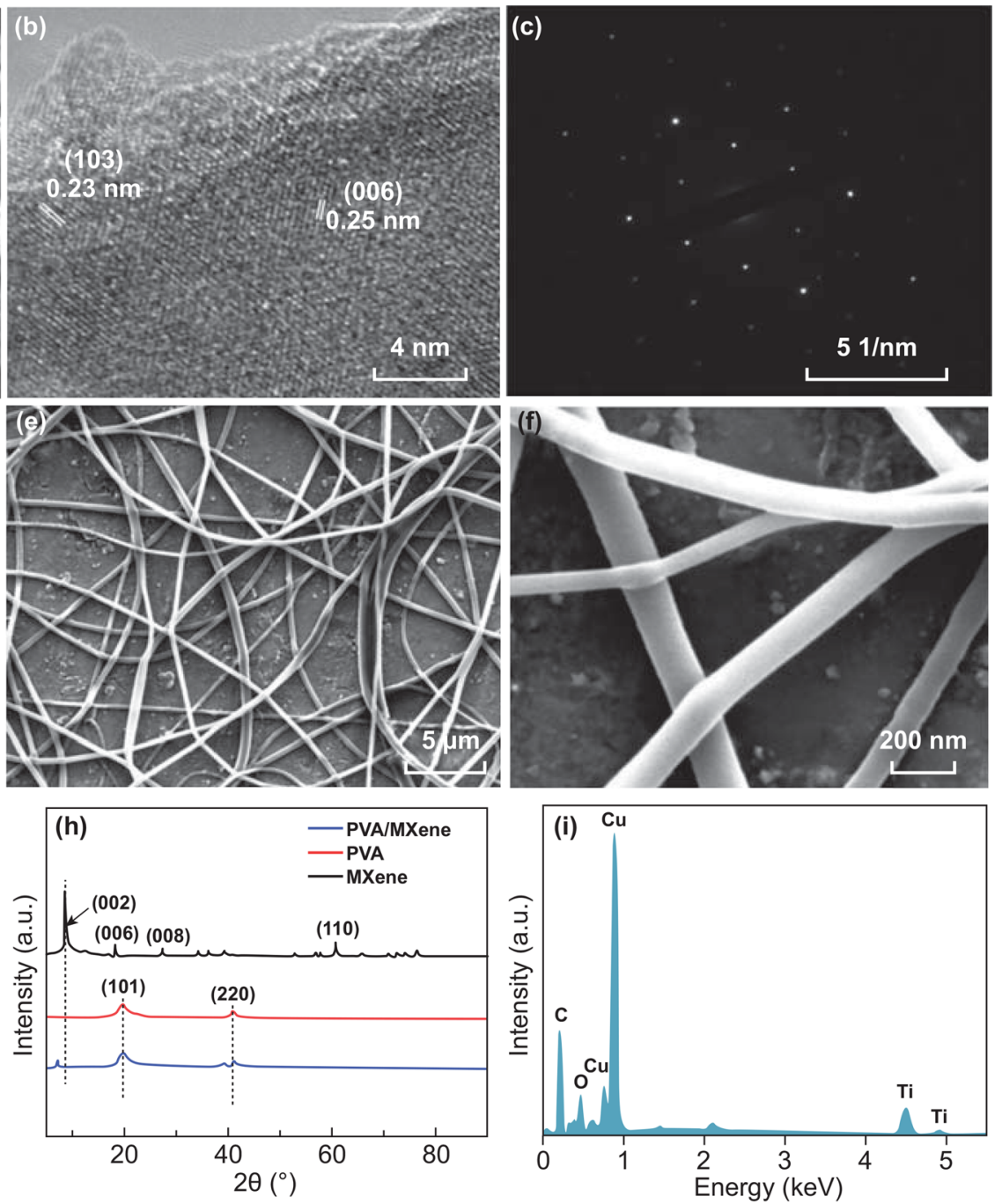
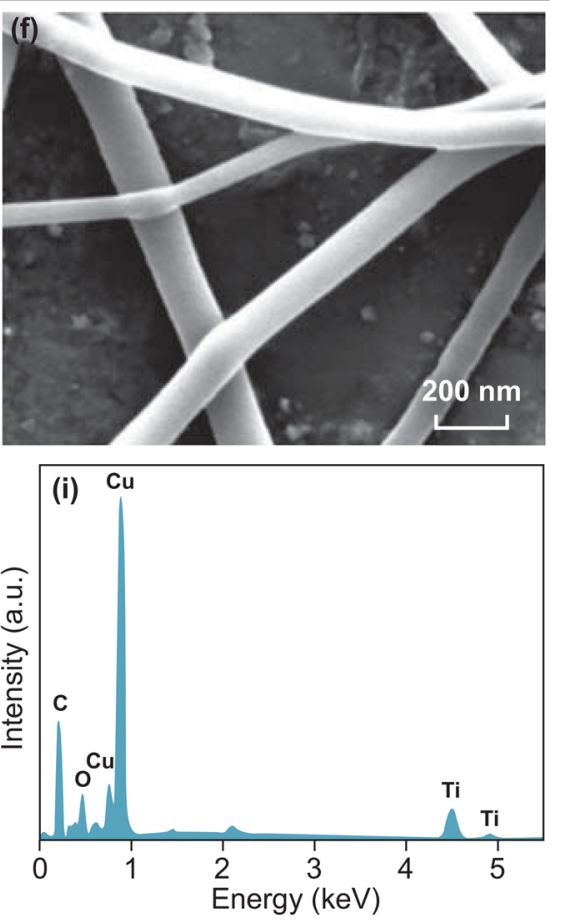

Fig. 4 a SEM of MXene multilayer structure. b TEM images of few-layer MXene nanoflakes. c Corresponding selected-area electron diffraction (SAED) pattern of the hexagonal arrangement of atoms. d, e SEM images of PVA/MXene nanofibers. $\mathbf{f}$ Contact angle measurement of MXene and PVA/MXene. $\mathbf{g}$ XRD, $\mathbf{h}$ EDS, and i FTIR pattern of MXene, PVA, and PVA/MXene composite 
$15^{\circ}, 30^{\circ}, 45^{\circ}$, and $75^{\circ}$. When the bending degree increases, the PENG exhibits an enhanced output voltage. The similar experiment was performed with the device attached to the wrist in Fig. 3b. The bending of the wrist produces a smaller output voltage compared to finger under the same angle. This could be because the knuckles cause a greater degree of bending of flexible PET. The device was placed on a sponge and applied different pressures with fingertip. Figure $3 \mathrm{c}$ shows the output voltage under different pressures. The pressure can be identified by detecting the output voltage. Figure $3 \mathrm{~d}$ shows that the device can identify swallowing action of the throat. And based on this, we detect the relative voltage change of $\mathrm{MoSe}_{2}$ flexible device by making different kinds of sounds, such as "nano," "energy," and "sensor" (Fig. 3e). As shown in Fig. 3f, we also detected the output voltage based on neck bending when the device was attached to the nape. Considering that neck diseases of an increasing number of teenagers due to looking down and playing with mobile phones, this application will have great prospects in the future. Human body is mainly driven by legs to walk and run. Figure $3 \mathrm{~g}-\mathrm{i}$ shows the energy harvesting when the device was attached to the knee and sole of the foot; the device can stably collect the energy generated by the legs and identify different movements like walking and running.

\subsection{Characterization of PEHS}

Figure 4a shows the SEM of MXene multilayer structure; the small particles between layers may be the broken MXene or $\mathrm{TiO}_{2}$ [43]. Figure 4b, c shows the TEM images of fewlayer MXene nanoflakes and the corresponding selectedarea electron diffraction (SAED) pattern of the hexagonal arrangement of atoms, respectively. The SAED exhibits the hexagonal arrangement of atoms. As shown in Fig. 4d-f, the SEM images show that the PVA/MXene nanofibers are successfully prepared. The average fiber diameter of PVA/ MXene nanofibers is $170 \mathrm{~nm}$. As shown in Fig. $2 \mathrm{~g}$, the water contact angles of MXene and PVA/MXene were $35.7^{\circ}$ and $24.5^{\circ}$, respectively. The PVA/MXene has the minimal contact angle and exhibits excellent hydrophilicity. The XRD characterization results of PVA, MXene, PVA/MXene are illustrated in Fig. 4h. The XRD pattern of MXene shows four prominent peaks at $2 \theta=8.4^{\circ}, 18.1^{\circ}, 26.8^{\circ}$, and $60.6^{\circ}$, which
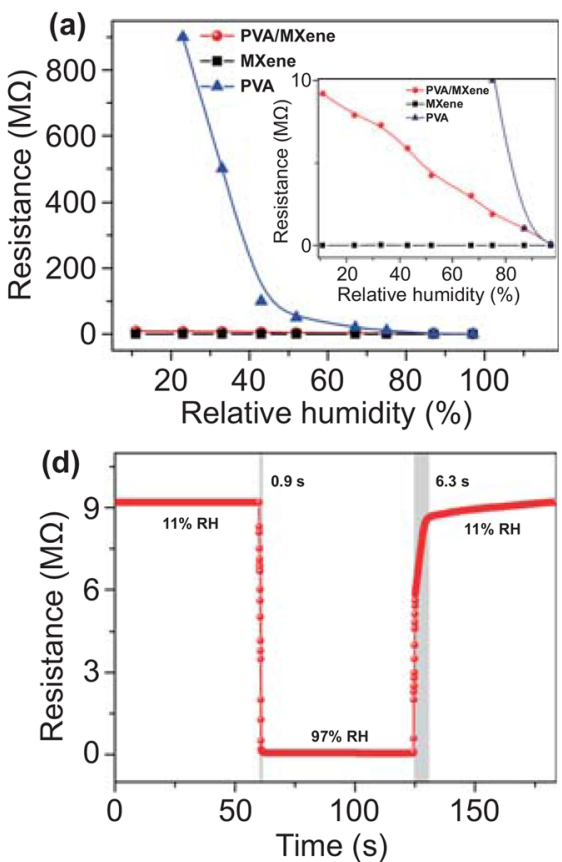
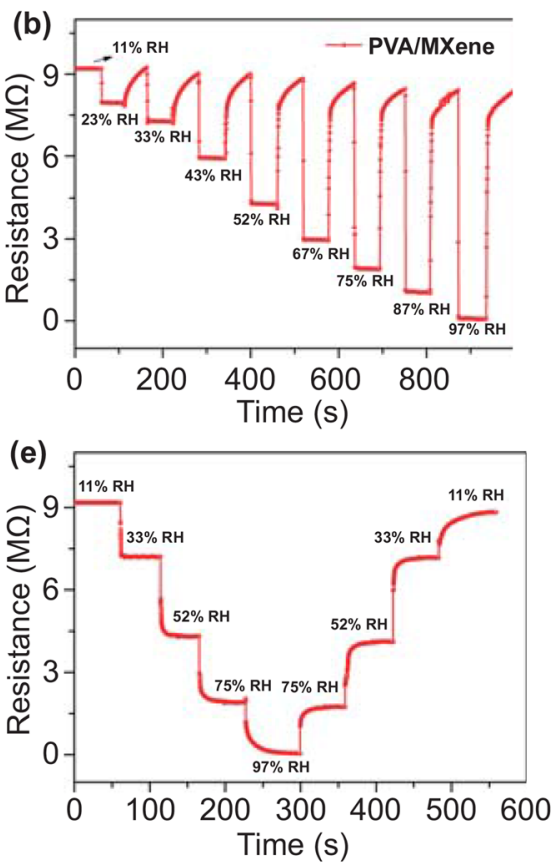
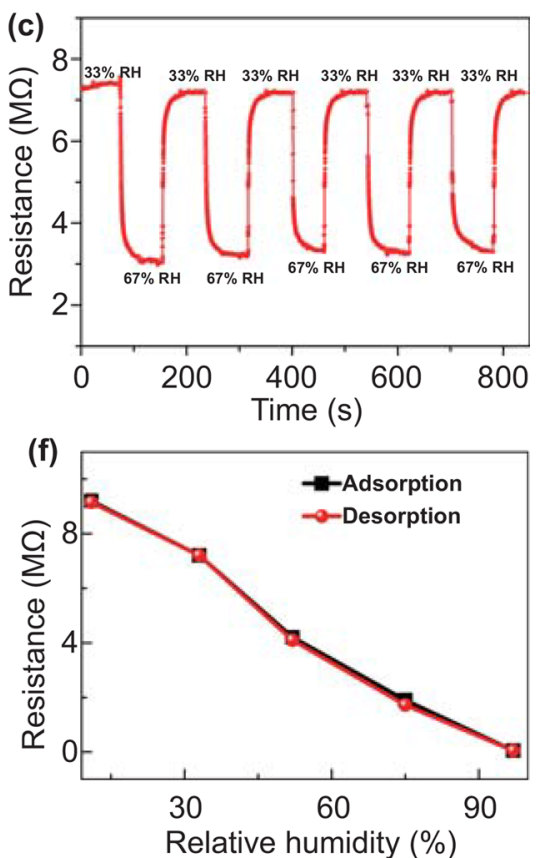

Fig. 5 a Resistance of the MXene, PVA, and PVA/MXene film sensor exposed to various relative humidities. b Dynamic resistance changes of PVA/MXene film sensor exposed to various relative humidities. c Repeatability of PVA/MXene film sensor. d Time-dependent resistance response and recovery curves of the PVA/MXene sensor between 11 and 97\% RH. e Resistance of sensor with increasing and decreasing humidity. f Humidity hysteresis curves of the PVA/MXene nanofibers film sensor 
are assigned to the (002), (006), (008), and (110) planes [32]. The XRD pattern of pure PVA shows two character peaks at $2 \theta=19.4^{\circ}$ and $41.2^{\circ}$, which are attributed to the (101) and (220) planes [44]. And it can be found that PVA did not destroy the crystal structure of MXene from the XRD pattern of PVA/MXene. The (002) peak for PVA/MXene is downshifted from $2 \theta=8.4^{\circ}$ to $2 \theta=7.2^{\circ}$ as compared to that of MXene; this change is considered to be due to the increase in the distance between the MXene nanosheets causing by the deposition of PVA molecules [36].

Figure 4i shows energy-dispersive spectrometer (EDS) images of PVA/MXene nanofibers film. The Ti and $\mathrm{O}$ elements are derived from MXene and PVA, respectively, and the $\mathrm{C}$ element comes from both MXene and PVA. The $\mathrm{Cu}$ is derived from copper foil substrate. The FTIR characterization results of PVA, MXene, PVA/MXene are illustrated in Fig. S4. The wide peak for all materials at about $3440 \mathrm{~cm}^{-1}$ is $\mathrm{O}-\mathrm{H}$ stretching vibration peak. The other typical peaks of PVA are at $2930 \mathrm{~cm}^{-1}$ for $\mathrm{C}-\mathrm{H}$ stretch, $1720 \mathrm{~cm}^{-1}$ for $\mathrm{C}=\mathrm{O}$ stretch, $1631 \mathrm{~cm}^{-1}$ for $-\mathrm{C}-\mathrm{C}-$ stretch, $1095 \mathrm{~cm}^{-1}$ for $\mathrm{C}-\mathrm{O}$ stretch, respectively [45]. The other typical peaks of MXene are observed at 1710 and $550 \mathrm{~cm}^{-1}$, which are assigned to the stretching vibration of $\mathrm{C}-\mathrm{O}$ and $\mathrm{O}-\mathrm{H}$, respectively [46].
The FTIR characterization result of PVA/MXene shows no obvious broad peak or shifts after the addition of MXene [47].

\subsection{Humidity Sensing Properties of PEHS}

The resistances of MXene, PVA, PVA/MXene sensors at different humidity levels are shown in Fig. 5a. Different humidity levels are provided by corresponding saturated salt solutions [48]. The resistance variation range (0.08-9.2 M $\Omega$ ) of $\mathrm{PVA} / \mathrm{MXene}$ sensor is aligned with the rising $U-R$ (voltageresistance) region of PENG (the U-R curve in Fig. $2 \mathrm{~g}$ ). The PVA sensor exhibited a high resistance state $(\geq 80 \mathrm{M} \Omega$ ) over a wide humidity range (11-52\% RH), and the MXene sensor showed a small resistance change (2.1-3.3 k $\Omega$ ), which are not conducive to the combination of the sensor and PENG as a self-powered humidity sensor. Therefore, PVA/MXene sensor driven by the prepared PENG is easier to obtain the corresponding relationship between output voltage and humidity levels. Figure $5 \mathrm{~b}$ shows the dynamic resistance change of the PVA/MXene sensor exposed to various RHs. The response/recovery time is defined as the time when the sensor achieved $90 \%$ of total resistance variation. Figure $5 \mathrm{c}$,
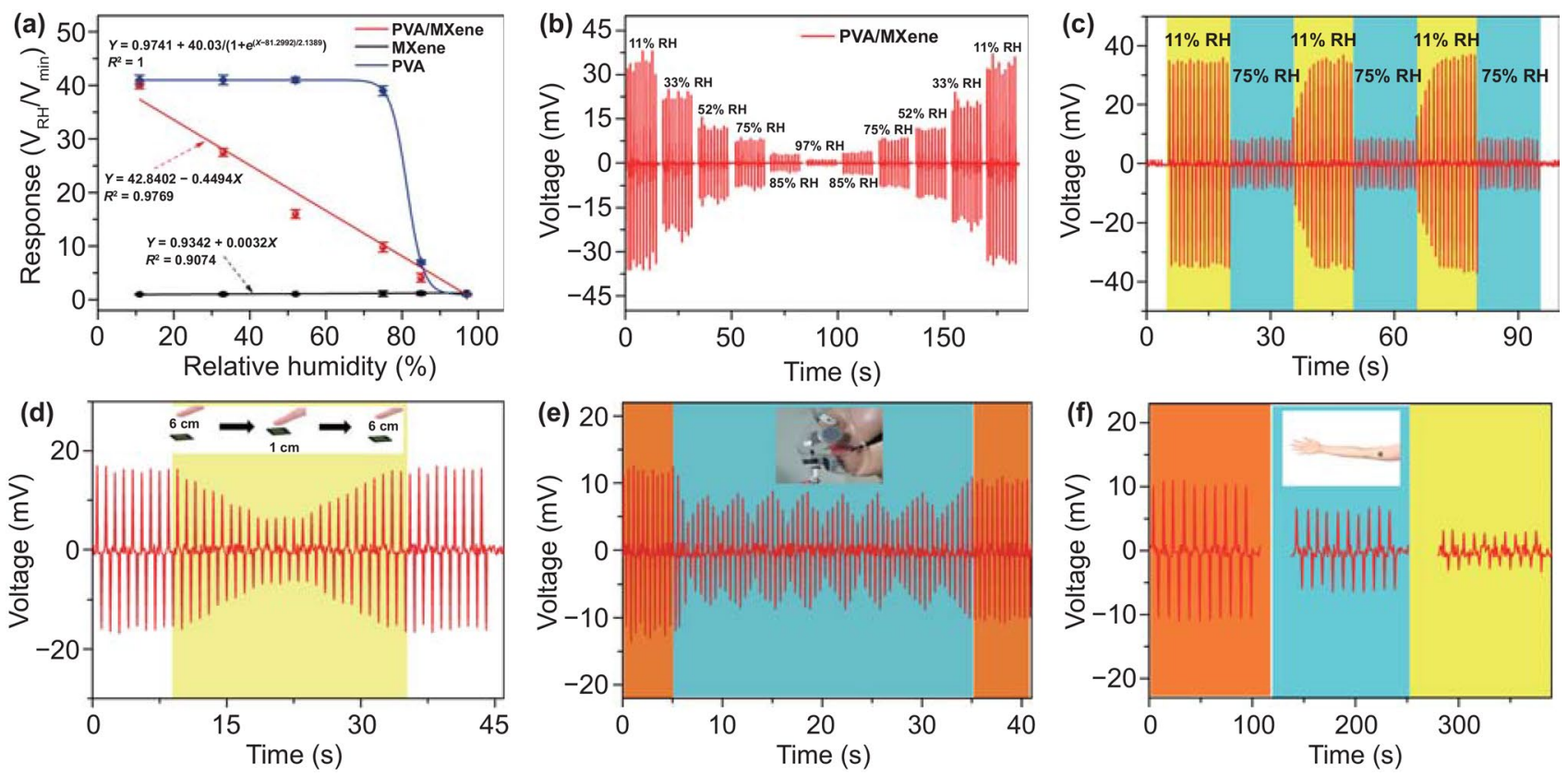

Fig. 6 a Response fitting curves of self-powered MXene, PVA, and PVA/MXene film sensors toward different humidities. b Output voltage for PVA/MXene-based PEHS upon exposure to different humidities. c Repeatability of self-powered PVA/MXene sensor. d Output voltage when the finger slowly approaches the sensor. e Flexible sensor is used to detect human breathing rate. $\mathbf{f}$ Test result of detecting the humidity of arm skin surface after different exercise times 
Table 2 Performance of the presented sensor in this work compared with the previous work

\begin{tabular}{llcll}
\hline Sensor materials & Meas. range & Response & Response/recovery time & Refs. \\
\hline RGO/PVP & $7-97.3 \% \mathrm{RH}$ & 7 & $2.8 / 3.5 \mathrm{~s}(90 \%)$ & \\
$\mathrm{LiCl}$ & $40-80 \% \mathrm{RH}$ & 12 & $\sim$ & {$[53]$} \\
$\mathrm{Ga} / \mathrm{ZnO}$ & $45-80 \% \mathrm{RH}$ & 4 & $5 \mathrm{~s}(90 \%)$ & {$[25]$} \\
PTFE/Al & $20-100 \% \mathrm{RH}$ & 28 & $18 / 80 \mathrm{~ms}(\sim)$ & {$[54]$} \\
PVA/MXene & $11-97 \% \mathrm{RH}$ & 40 & $0.9 / 6.3 \mathrm{~s}(90 \%)$ & {$[24]$} \\
\hline
\end{tabular}

d shows that the sensor has good repeatability and fast response/recovery time (0.9/6.3 s), respectively. As shown in Fig. 5e, f, we investigated the hysteresis characteristic of PVA/MXene sensor versus RH. The sensor hysteresis is defined as $\mathrm{H}=\left(R_{\mathrm{A}}-R_{\mathrm{D}}\right) / S(\% \mathrm{RH}) . R_{\mathrm{A}}$ and $R_{\mathrm{D}}$ are the sensor resistance in the adsorption and desorption process of water molecules, and $\mathrm{S}$ is the sensor sensitivity. We investigated the hysteresis characteristic of PVA/MXene sensor versus $\mathrm{RH}$, which shows the PVA/MXene humidity sensor has low hysteresis of $1.8 \%$. The sensor has excellent humidity sensing performance, which is attributed to the humidity sensitivity of composite material. MXene has strong hydrophilicity and high electrical conductivity. The resistance of MXene film increases with the increase in humidity level, which may be the result of the increase in layer spacing caused by water molecules embedded in the MXene layers $[49,50]$. The conductivity change of PVA/MXene can be caused by the adsorption of water molecules under humidity environment. Both PVA and MXene contain a large number of hydroxyl groups $(-\mathrm{OH})$, and the proton can transition between two adjacent hydroxyl groups. In addition, protons can help electron transfer between water molecules. Under low humidity level, proton-assisted electron tunneling is the main reaction process. Under high humidity level, water molecules are firmly bound to hydroxyl groups. Hydrogen ions formed by PVA adsorbed water molecules hop between water molecules. With the increase in humidity, the concentration of hydrogen ions increases, resulting in the decrease in sensor resistance. In addition, MXene can be used as the charge transmission and conduction layer of composite materials due to its excellent metallic conductivity, which is conducive to accelerating the adsorption/desorption process of water molecules. Therefore, the PVA/MXene sensor achieved a fast response/recovery behavior [51, 52].

Figure 6a shows the response-humidity fitting curves of MXene, PVA, and PVA/MXene sensors at 11-97\% RH. The actual voltage value is shown in Fig. S3a. The minimum value of output voltage is the reference value of the sensor response. The response of humidity sensor was defined as: $S=V_{\mathrm{RH}} / V_{\min }$, where the $V_{\mathrm{RH}}$ is the output voltage of sensor at the target humidity and the $V_{\min }$ is the minimum output voltage. The PVA sensor has the same voltage output in the humidity range of $11-52 \%$ because of its large resistance, and the voltage output of MXene sensor is close to 0 because of its small resistance. The experimental results are consistent with the previous analysis results; neither PVA nor MXene sensor is suitable to combine with PENG to detect humidity. The prepared self-powered PVA/MXene sensor holds high humidity response of $\sim 40$. The corresponding equation is $\mathrm{Y}=42.8402-0.4494 \mathrm{X}$, and the regression coefficient $\left(R^{2}\right)$ is 0.9769 . Figure $6 \mathrm{~b}$ shows the output voltage for PVA/MXene nanofibers film sensor driven by PENG when sensor exposed to wide humidity range (11-97\% RH). It can be seen that the output voltage exhibits highest value at $11 \%$ $\mathrm{RH}$ and has obvious decrease with increasing humidity. The different humidity levels can be distinguished by particular output voltage. The self-powered PVA/MXene nanofiber film sensor has excellent repeatability as in Fig. 6c. There is no obvious change in output voltage by comparing test result. And we also measured the output voltage when the finger slowly approaches the sensor as in Fig. 6d. Finger approaches the sensor at a constant speed $\left(0.5 \mathrm{~cm} \mathrm{~s}^{-1}\right)$ at a distance from the sensor $(6 \mathrm{~cm})$ and then leaves at the same speed. Table 2 summarizes the humidity sensing performance of the presented PEHS in comparison with previous works [24, 25, 53, 54]; the comparison highlights the PEHS has much higher response in a wide RH range. Figure S3b shows the long-term stability of PVA/MXene nanofibers film sensor driven by PENG over a period of 30 days. It can be found that the sensor has no noticeable voltage drift and exhibits excellent stability. Figure 6e shows the output voltage when the flexible sensor is used to detect human breathing rate. Figure $6 f$ shows the test result of detecting the humidity of arm skin surface after different exercise times 
when the sensor is attached to the arm. The output voltages all occur regular changes. Thus, the flexible humidity sensor driven by PENG exhibits excellent performance on detecting human skin surface moisture and has great application prospects in wearable devices.

\section{Conclusions}

In this work, a self-powered humidity sensing device based on monolayer $\mathrm{MoSe}_{2}$ PENG has been proposed for the detection of humidity. The piezoelectric properties of monolayer layer $\mathrm{MoSe}_{2}$ were reported at first time. A high peak output of $35 \mathrm{mV}$ can be obtained when the PENG was under $0.36 \%$ strain at a frequency of $0.5 \mathrm{~Hz}$. And the flexible PENG can harvest energy and generate different output voltages by attaching to different parts of human body. The self-powered sensor was prepared by PVA/MXene composite nanofibers film and driven by the monolayer $\mathrm{MoSe}_{2}$ PENG to detect humidity by converting mechanical energy to electric energy, which has a larger response (40) and 40-fold higher than pure MXene. And the humidity sensor also shows fast response/recovery time of $0.9 / 6.3 \mathrm{~s}$, low hysteresis of $1.8 \%$, and stable repeatability. Moreover, the PVA/MXene nanofibers film was also used to prepare flexible humidity sensor on a PET flexible substrate and exhibited excellent performance on detecting human skin surface moisture.

Acknowledgements This work was supported by the National Natural Science Foundation of China (51777215), National Natural Science Foundation of China (51775306), Beijing Municipal Natural Science Foundation (4192027), and the Graduate Innovation Fund of China University of Petroleum (YCX2020097).

Open Access This article is licensed under a Creative Commons Attribution 4.0 International License, which permits use, sharing, adaptation, distribution and reproduction in any medium or format, as long as you give appropriate credit to the original author(s) and the source, provide a link to the Creative Commons licence, and indicate if changes were made. The images or other third party material in this article are included in the article's Creative Commons licence, unless indicated otherwise in a credit line to the material. If material is not included in the article's Creative Commons licence and your intended use is not permitted by statutory regulation or exceeds the permitted use, you will need to obtain permission directly from the copyright holder. To view a copy of this licence, visit http://creativecommons.org/licenses/by/4.0/.
Supplementary Information The online version contains supplementary material available at(https://doi.org/10.1007/s4082 0-020-00580-5)

\section{References}

1. J. Cai, C. Lv, E. Aoyagi, S. Ogawa, A. Watanabe, Laser direct writing of a high-performance all-graphene humidity sensor working in a novel sensing mode for portable electronics. ACS Appl. Mater. Interfaces 10, 23987-23996 (2018). https://doi. org/10.1021/acsami.8b07373

2. B. Li, Q. Tian, H. Su, X. Wang, T. Wang et al., High sensitivity portable capacitive humidity sensor based on $\mathrm{In}_{2} \mathrm{O}_{3}$ nanocubesdecorated GO nanosheets and its wearable application in respiration detection. Sens. Actuat. B 299, 126973 (2019). https:// doi.org/10.1016/j.snb.2019.126973

3. D. Zhing, D. Wang, P. Li, X. Zhou, X. Zong et al., Facile fabrication of high-performance QCM humidity sensor based on layer-by-layer self-assembled polyaniline/graphene oxide nanocomposite film. Sens. Actuat. B 255, 1869-1877 (2018). https://doi.org/10.1016/j.snb.2017.08.212

4. Y. Yao, Y. Xue, Influence of the oxygen content on the humidity sensing properties of functionalized graphene films based on bulk acoustic wave humidity sensors. Sens. Actuat. B 222, 755-762 (2016). https://doi.org/10.1016/j.snb.2015.08.121

5. Z. Xu, Y. Yuan, Implementation of guiding layers of surface acoustic wave devices: a review. Biosens. Bioelectron. 99, 500-512 (2018). https://doi.org/10.1016/j.bios.2017.07.060

6. Y. Yang, J. Qi, Q. Liao, H. Li, Y. Wang et al., High-performance piezoelectric gate diode of a single polar-surface dominated ZnO nanobelt. Nanotechnology 20, 125201 (2009). https://doi. org/10.1088/0957-4484/20/12/125201

7. Y. Yang, J. Qi, W. Guo, Y. Gu, Y. Huang et al., Transverse piezoelectric field-effect transistor based on single $\mathrm{ZnO}$ nanobelts. Phys. Chem. Chem. Phys. 12, 12415-12419 (2019). https ://doi.org/10.1039/C0CP00420K

8. T. Gao, Y. Ji, Y. Yang, Thermo-phototronic effect induced electricity in long semiconducting $\mathrm{ZnO}$ materials for self-powered light and temperature sensors. Adv. Mater. Technol. US 5(7), 2000176 (2020). https://doi.org/10.1002/admt.202000176

9. Y. Yang, W. Guo, J. Qi, Y. Zhang, Flexible piezoresistive strain sensor based on single Sb-doped $\mathrm{ZnO}$ nanobelts. Appl. Phys. Lett. 97, 223107 (2010). https://doi.org/10.1063/1.3522885

10. D. Zhang, K. Zhang, Y. Wang, Y. Wang, Y. Yang, Thermoelectric effect induced electricity in stretchable graphenepolymer nanocomposites for ultrasensitive self-powered strain sensor system. Nano Energy 56, 25-32 (2019). https://doi. org/10.1016/j.nanoen.2018.11.026

11. Y. Wang, Y. Wang, Y. Yang, Graphene-polymer nanocompositebased redox-induced electricity for flexible self-powered strain sensors. Adv. Energy Mater. 8, 1800961 (2018). https://doi. org/10.1002/aenm.201800961 
12. K. Zhang, S. Wang, Y. Yang, A one-structure-based piezotribo-pyro-photoelectric effects coupled nanogenerator for simultaneously scavenging mechanical, thermal, and solar energies. Adv. Energy Mater. 7, 1601852 (2017). https://doi. org/10.1002/aenm.201601852

13. W. Qian, W. Yang, Y. Zhang, C.R. Bowen, Y. Yang, Piezoelectric materials for controlling electro-chemical processes. Nano-Micro Lett. 12, 149 (2020). https://doi.org/10.1007/s4082 0-020-00489-z

14. K. Zhao, Z.L. Wang, Y. Yang, Self-powered wireless smart sensor node enabled by an ultrastable, highly efficient, and superhydrophobic-surface-based triboelectric nanogenerator. ACS Nano 10, 9044-9052 (2016). https://doi.org/10.1021/acsna no. $6 \mathrm{~b} 05815$

15. H. Wang, Z. Xiang, P. Giorgia, X. Mu, Y. Yang et al., Triboelectric liquid volume sensor for self-powered lab-on-chip applications. Nano Energy 23, 80-88 (2016). https://doi.org/10.1016/j. nanoen.2016.02.054

16. Z. Wu, W. Ding, Y. Dai, K. Dong, C. Wu et al., Self-powered multifunctional motion sensor enabled by magnetic-regulated triboelectric nanogenerator. ACS Nano 12, 5726-5733 (2018). https://doi.org/10.1021/acsnano.8b01589

17. Z. Wu, B. Zhang, H. Zou, Z. Lin, G. Liu et al., Multifunctional sensor based on translational-rotary triboelectric nanogenerator. Adv. Energy Mater. 9, 1901124 (2019). https://doi.org/10.1002/ aenm.201901124

18. K. Song, N. Ma, Y.K. Mishra, R. Adelung, Y. Yang, Achieving light-induced ultrahigh pyroelectric charge density toward selfpowered UV light detection. Adv. Electron. Mater. 5, 1800413 (2019). https://doi.org/10.1002/aelm.201800413

19. K. Zhao, B. Ouyang, C.R. Bowen, Z.L. Wang, Y. Yang, Onestructure-based multi-effects coupled nanogenerators for flexible and self-powered multi-functional coupled sensor systems. Nano Energy 71, 104632 (2020). https://doi.org/10.1016/j. nanoen.2020.104632

20. Y. Chen, Y. Cheng, Y. Jie, X. Cao, N. Wang et al., Energy harvesting and wireless power transmission by a hybridized electromagnetic-triboelectric nanogenerator. Energy Environ. Sci. 12, 2678-2684 (2019). https://doi.org/10.1039/C9EE01245A

21. Z. Wu, T. Cheng, Z.L. Wang, Self-powered sensors and systems based on nanogenerators. Sensors 20, 2925 (2020). https://doi. org/10.3390/s20102925

22. Z.L. Wang, J. Song, Piezoelectric nanogenerators based on zinc oxide nanowire arrays. Science 312, 242-246 (2006). https:// doi.org/10.1126/science. 1124005

23. C. Sun, Q. Shi, D. Hasan, M.S. Yazici, M. Zhu et al., Self-powered multifunctional monitoring system using hybrid integrated triboelectric nanogenerators and piezoelectric microsensors. Nano Energy 58, 612-623 (2019). https://doi.org/10.1016/j. nanoen.2019.01.096

24. M. Ma, Q. Liao, G. Zhang, Z. Zhang, Q. Liang et al., Selfrecovering triboelectric nanogenerator as active multifunctional sensors. Adv. Funct. Mater. 25, 6489-6494 (2015). https://doi. org/10.1002/adfm.201503180

25. K. Xia, Z. Zhu, J. Fu, Y. Chi, Z. Xu, Multifunctional conductive copper tape-based triboelectric nanogenerator and as a self-powered humidity sensor. IEEE Trans. Electron Devices 66, 2741-2745 (2019). https://doi.org/10.1109/TED.2019.2911637

26. S. Wang, H. Tai, B. Liu, Z. Duan, Z. Yuan et al., A facile respiration-driven triboelectric nanogenerator for multifunctional respiratory monitoring. Nano Energy 58, 312-321 (2019). https ://doi.org/10.1016/j.nanoen.2019.01.042

27. K.A.N. Duerloo, M.T. Ong, E.J. Reed, Intrinsic piezoelectricity in two-dimensional materials. J. Phys. Chem. Lett. 3, 28712876 (2012). https://doi.org/10.1021/jz3012436

28. W. Wu, L. Wang, Y. Li, F. Zhang, L. Lin et al., Piezoelectricity of single-atomic-layer $\mathrm{MoS}_{2}$ for energy conversion and piezotronics. Nature 514, 470-474 (2014). https://doi.org/10.1038/ nature 13792

29. D. Zhang, Z. Yang, P. Li, M. Pang, Q. Xue, Flexible self-powered high-performance ammonia sensor based on Au-decorated $\mathrm{MoSe}_{2}$ nanoflowers driven by single layer $\mathrm{MoS}_{2}$-flake piezoelectric nanogenerator. Nano Energy 65, 103974 (2019). https://doi. org/10.1016/j.nanoen.2019.103974

30. E. Lee, A. Vahidmohammadi, B.C. Prorok, Y.S. Yoon, M. Beidaghi et al., Room temperature gas sensing of two-dimensional titanium carbide (MXene). ACS Appl. Mater. Interfaces 9, 37184 37190 (2017). https://doi.org/10.1021/acsami.7b11055

31. Y. Yue, N. Liu, W. Liu, M. Li, Y. Ma et al., 3D hybrid porous Mxene-sponge network and its application in piezoresistive sensor. Nano Energy 50, 79-87 (2018). https://doi.org/10.1016/j. nanoen.2018.05.020

32. M. Naguib, M. Kurtoglu, V. Presser, J. Lu, J. Niu et al., Twodimensional nanocrystals produced by exfoliation of $\mathrm{Ti}_{3} \mathrm{AlC}_{2}$. Adv. Mater. 23, 4248-4253 (2011). https://doi.org/10.1002/ adma.201102306

33. Z. Yang, A. Liu, C. Wang, F. Liu, J. He et al., Improvement of gas and humidity sensing properties of organ-like MXene by alkaline treatment. ACS Sens. 4, 1261-1269 (2019). https://doi. org/10.1021/acssensors.9b00127

34. K. Wang, Z. Lou, L. Wang, L. Zhao, S. Zhao et al., Bioinspired interlocked structure-induced high deformability for two-dimensional titanium carbide (MXene)/natural microcapsule-based flexible pressure sensors. ACS Nano 13, 9139-9147 (2019). https:// doi.org/10.1021/acsnano.9b03454

35. R. Yang, J. He, L. Xu, J. Yu, Effect of solution concentration on diameter and morphology of PVA nanofibres in bubble electrospinning process. J. Mater. Sci. Technol. 26, 1313-1316 (2010). https://doi.org/10.1179/026708310X12798718274476

36. C. Jiang, C. Wu, X. Li, Y. Yao, L. Lan et al., All-electrospun flexible triboelectric nanogenerator based on metallic MXene nanosheets. Nano Energy 59, 268-276 (2019). https://doi. org/10.1016/j.nanoen.2019.02.052

37. X. Chen, P. Hu, K. Song, X. Wang, C. Zuo et al., CVD growth of large-scale hexagon-like shaped $\mathrm{MoSe}_{2}$ monolayers with sawtooth edge. Chem. Phys. Lett. 733, 136663 (2019). https://doi. org/10.1016/j.cplett.2019.136663

38. Y. Chang, W. Zhang, Y. Zhu, Y. Han, J. Pu et al., Monolayer $\mathrm{MoSe}_{2}$ grown by chemical vapor deposition for fast photodetection. ACS Nano 8, 8582-8590 (2014). https://doi.org/10.1021/ nn503287m 
39. P. He, W. Chen, J. Li, H. Zhang, Y. Li et al., Keggin and Dawson polyoxometalates as electrodes for flexible and transparent piezoelectric nanogenerators to efficiently utilize mechanical energy in the environment. Sci. Bull. 65, 35-44 (2020). https:// doi.org/10.1016/j.scib.2019.09.026

40. G. Viola, J. Chang, T. Maltby, F. Steckler, M. Jomaa et al., Bioinspired multiresonant acoustic devices based on electrospun piezoelectric polymeric nanofibers. ACS Appl. Mater. Interfaces 12, 34643-34657 (2020). https://doi.org/10.1021/acsami.0c09238

41. P.K. Yang, S.A. Chou, C.H. Hsu, R.J. Mathew, K.H. Chiang et al., Tin disulfide piezoelectric nanogenerators for biomechanical energy harvesting and intelligent human-robot interface applications. Nano Energy 75, 104879 (2020). https://doi.org/10.1016/j. nanoen.2020.104879

42. Z. Wang, H. Kurita, H. Nagaoka, F. Narita, Potassium sodium niobate lead-free piezoelectric nanocomposite generators based on carbon-fiber-reinforced polymer electrodes for energy-harvesting structures. Compos. Sci. Technol. 199, 108331 (2020). https://doi. org/10.1016/j.compscitech.2020.108331

43. C. Peng, X. Yang, Y. Li, H. Yu, H. Wang et al., Hybrids of two-dimensional $\mathrm{Ti} 3 \mathrm{C} 2$ and $\mathrm{TiO} 2$ exposing 001 facets toward enhanced photocatalytic activity. ACS Appl. Mater. Interfaces 8 , 6051-6060 (2016). https://doi.org/10.1021/acsami.5b11973

44. M. Das, D. Sarkar, Development of room temperature ethanol sensor from polypyrrole (PPy) embedded in polyvinyl alcohol (PVA) matrix. Polym. Bull. 75, 3109-3125 (2018). https://doi. org/10.1007/s00289-017-2192-y

45. J. Fan, J. Luo, X. Zhang, B. Zhen, C. Dong et al., A novel electrospun $\beta$-CD/CS/PVA nanofiber membrane for simultaneous and rapid removal of organic micropollutants and heavy metal ions from water. Chem. Eng. J. 378, 122232 (2019). https://doi. org/10.1016/j.cej.2019.122232

46. L. Wang, L. Chen, P. Song, C. Liang, Y. Lu et al., Fabrication on the annealed $\mathrm{Ti}_{3} \mathrm{C}_{2} \mathrm{~T}_{\mathrm{x}}$ MXene/Epoxy nanocomposites for electromagnetic interference shielding application. Compos Part B 171, 111-118 (2019). https://doi.org/10.1016/j.composites b.2019.04.050
47. E.A. Mayerberger, O. Urbanek, R.M. McDaniel, R.M. Street, M.W. Barsoum et al., Preparation and characterization of polymer- $\mathrm{Ti}_{3} \mathrm{C}_{2} \mathrm{~T}_{\mathrm{x}}$ (MXene) composite nanofibers produced via electrospinning. J. Appl. Polym. Sci. 134, 45295 (2017). https://doi. org/10.1002/app.45295

48. D. Zhang, D. Wang, X. Zong, G. Dong, Y. Zhang, High-performance QCM humidity sensor based on graphene oxide/tinoxide/ polyaniline ternary nanocomposite prepared by in-situoxidative polymerization method. Sens. Actuat. B 262, 531-541 (2018). https://doi.org/10.1016/j.snb.2018.02.012

49. H. An, T. Habib, S. Shah, H. Gao, A. Patel et al., Water Sorption in MXene/polyelectrolyte multilayers for ultrafast humidity sensing. ACS Appl. Nano Mater. 2, 948-955 (2019). https://doi. org/10.1021/acsanm.8b02265

50. E.S. Muckley, M. Naguib, H.W. Wang, L. Vlcek, N.C. Osti et al., Multimodality of structural, electrical, and gravimetric responses of intercalated MXenes to water. ACS Nano 11, 11118-11128 (2017). https://doi.org/10.1021/acsnano.7b05264

51. M. Naguib, V.N. Mochalin, M.W. Barsoum, Y. Gogotsi, 25th anniversary article: Mxenes: a new family of two-dimensional materials. Adv. Mater. 26, 992-1005 (2014). https://doi.org/10.1002/ adma.201304138

52. Z. Ahmad, M. Abbas, I. Gunawan, R.A. Shakoor, F. Ubaid et al., Electro-sprayed PVA coating with texture-enriched surface morphology for augmented humidity sensing. Prog. Org. Coat. 17, 7-9 (2018). https://doi.org/10.1016/j.porgcoat.2017.12.010

53. Y. Su, G. Xie, S. Wang, H. Tai, Q. Zhang et al., Novel highperformance self-powered humidity detection enabled by triboelectric effect. Sens. Actuat. B 251, 144-152 (2017). https://doi. org/10.1016/j.snb.2017.04.039

54. T. Zhao, Y. Fu, Y. Zhao, L. Xing, X. Xue, Ga-doped ZnO nanowire nanogenerator as self-powered/active humidity sensor with high sensitivity and fast response. J. Alloys Compd. 648, 571-576 (2015). https://doi.org/10.1016/j.jallcom.2015.07.035 